\title{
Diseño para la sostenibilidad en el coche eléctrico. Del Milburn Model 22 "Brougham" de 1916 al Renault Zoe de 2019
}

Jesús Jiménez Galea ${ }^{1}$ Universidad de Málaga

\section{Resumen}

Históricamente la humanidad ha mejorado el diseño de todo lo que ha ido construyendo, ya sea por mejorar su funcionalidad o embellecer su aspecto. Hoy día se necesita mejorar el diseño para a su vez mejorar la sostenibilidad. Un ejemplo claro de esta necesidad se tiene con los vehículos de combustión interna alternativa, que expulsan gases contaminantes a la atmósfera como el NOx del que se habla en el "Air Quality in Europe 2018" de la AEMA (Agencia Europea Medio Ambiente), y una de las soluciones que se está adoptando es mejorar el diseño de los vehículos para entre otras alternativas, electrificarlos.

Este artículo muestra cómo se ha adaptado el diseño de los vehículos eléctricos para mejorar su sostenibilidad y funcionalidad desde un vehículo construido en el año 1916 hasta un vehículo actual de 2019.

Palabras clave: sostenibilidad, diseño, automóvil, vehículo eléctrico.

Design for sustainability in the electric car.

From the Milburn Model 22 "Brougham" of 1916 to the Renault Zoe of 2019

\section{Abstract}

Historically, humanity has improved the design of everything it has built, either by improving its functionality or beautifying its appearance. Nowadays, it is necessary to improve the design in order to improve sustainability. A clear example is the alternative internal combustion vehicles, which expel pollutant gases into the atmosphere such as NOx which is spoken in the EEA (European Environment Agency) in its "Air Quality in Europe 2018", and one of the solutions being adopted is to improve the design of vehicles to, among other alternatives, electrify them.

This article shows how the design of electric vehicles has been adapted to improve its sustainability and functionality from a vehicle built in 1916 to a current vehicle of 2019.

Keywords: sustainability, design, car, electric vehicle.

Profesor en el Área de Ingeniería de los Procesos de Fabricación del Departamento de Ingeniería Civil de Materiales y Fabricación de la Universidad de Málaga. Doctorando en el Programa de Ingeniería Mecánica y Eficiencia Energética de la Universidad de Málaga. Ingeniero Técnico Industrial especialidad Mecánica, Ingeniero Técnico en Diseño Industrial, Experto Universitario en Nuevas Tecnologías en Sistemas de Propulsión para Vehículos, Máster Oficial en Prevención de Riesgos Laborales, Máster Propio Universitario en Innovación Educativa y Gestión del Conocimiento. Agradecer al Museo del Automóvil de Málaga y a Renault Tahermo su amabilidad y predisposición a la ayuda en este artículo.

Correo: j.jimenez@uma.es. 
La diferencia que suponen esos 103 años entre el Milburn Model 22 "Brougham" que se ve en la (fig. 1) y el Renault Zoe de la (fig. 2), se aprecia simplemente viendo el diseño exterior de ambos, y claro está, la evolución existente en el diseño interno y las mejoras tecnológicas, así como de habitabilidad, hacen que se entienda perfectamente el salto cualitativo entre ambos vehículos.

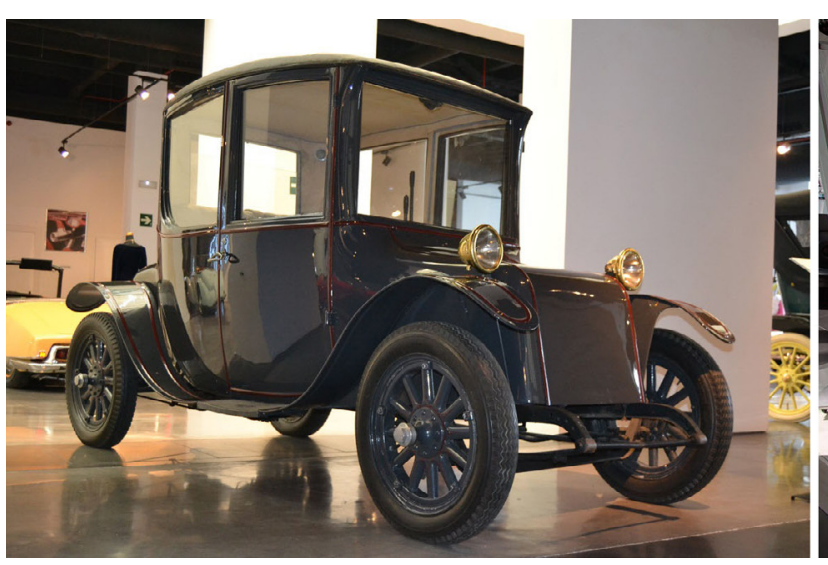

Fig. 1. Milburn Model 22 "Brougham”. JJJG.

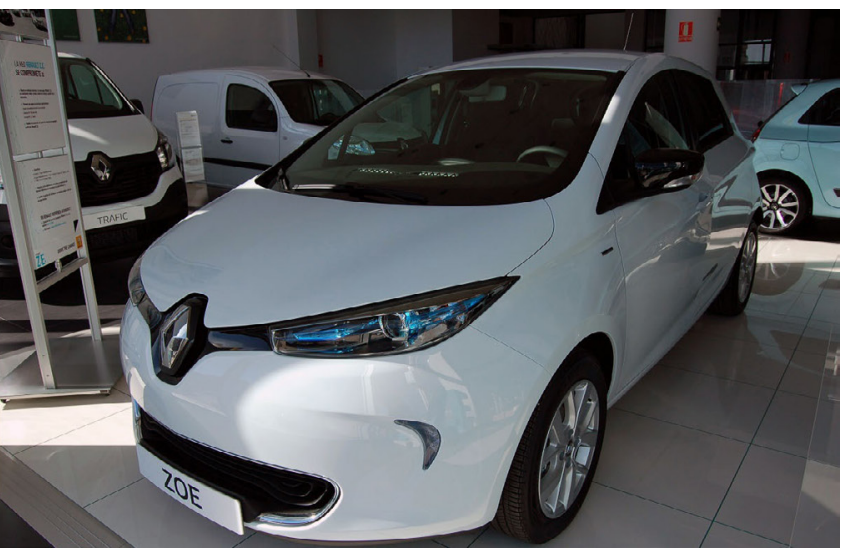

Fig. 2. Renault Zoe. JJJG.

Los fabricantes de vehículos, hoy día buscan tener una RSC (Responsabilidad Social Corporativa) lo más fuerte posible para que repercuta en una imagen positiva sobre el cliente potencial, sea un incentivo para comprar su producto, consiga una fidelización del cliente ${ }^{2}$ para su marca e incluso mejore las exportaciones ${ }^{3}$ en un mundo tan globalizado. Una manera de hacerlo es teniendo menos impacto para el planeta, tanto en la fabricación de los vehículos como disminuyendo la contaminación que los propios vehículos generan al medio ambiente. Esta manera de buscar la mejora de la imagen de la empresa o la institución preocupándose del medio ambiente y de los ciudadanos, dista mucho con lo que había hace un siglo, en la que el sistema que había incluso quería que el salario del trabajador se regulara según la oferta y la demanda dependiendo del número de trabajadores existentes ${ }^{4}$

2 McWilliams, A. y Siegel, D. S. (2011). Creating and capturing value: Strategic corporate social responsibility, resource-based theory, and sustainable competitive advantage. Journal of Management, 37 (5). 1480-1495.

3 Boehe, D.M. y Cruz, L.B. (2010). Corporate social responsibility, product differentiation strategy and export performance. Journal of Business Ethics, Vol. 91 No. 2, pp. 325-346.

4 París Eguilaz, H. (1941). Un Nuevo Orden Económico. Madrid, España: Ediciones FE, p. 27. 
Es por esto que resulta imprescindible disminuir el consumo de combustibles derivados del petróleo para bajar la contaminación en el planeta, estando muy agudizada en zonas industriales y grandes urbes como por ejemplo Madrid. Una opción para conseguir esto es el aumento del uso del vehículo eléctrico.

Actualmente el eléctrico más vendido en España es el Renault Zoe ${ }^{5}$, y lo es por derecho propio. El diseño exterior que tiene atrae las miradas, y a que Renault ha conseguido aunar armonía y belleza en este modelo, donde los faros delanteros y traseros dejan un sello inconfundible, pudiéndose ver el faro delantero derecho (fig. 3), y el faro trasero izquierdo (fig. 4). En el Milburn, los faros también eran en su época innovadores en diseño y eficiencia, tal y como se puede apreciar en la fig. 5. En la (fig. 6) se ve el testimonial faro trasero. La importancia de ser visto en carretera en aquellos años no era tan importante, ya que el parque automovilístico que había no es el que hay hoy día, y se primaba el que el conductor tuviera una buena visibilidad por encima de que se le viera.

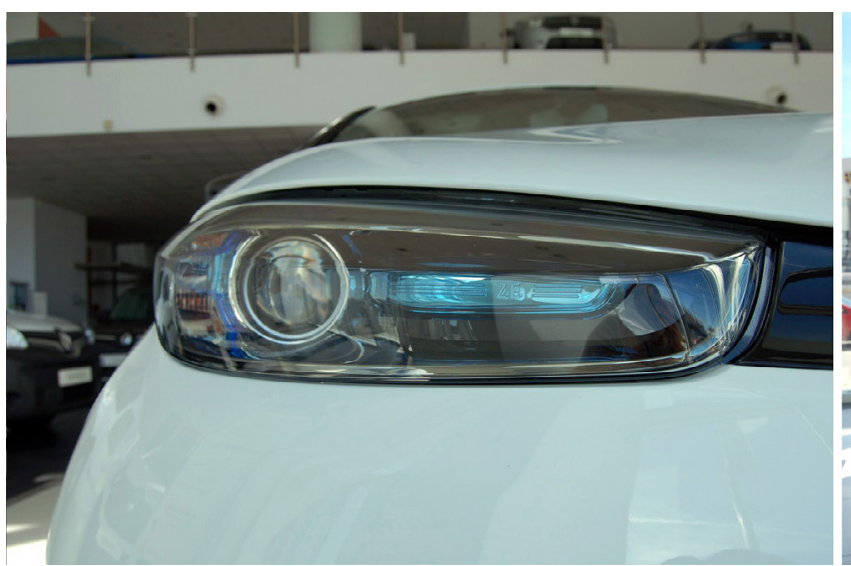

Fig. 3. Faro delantero derecho del Renault Zoe. JJJG.

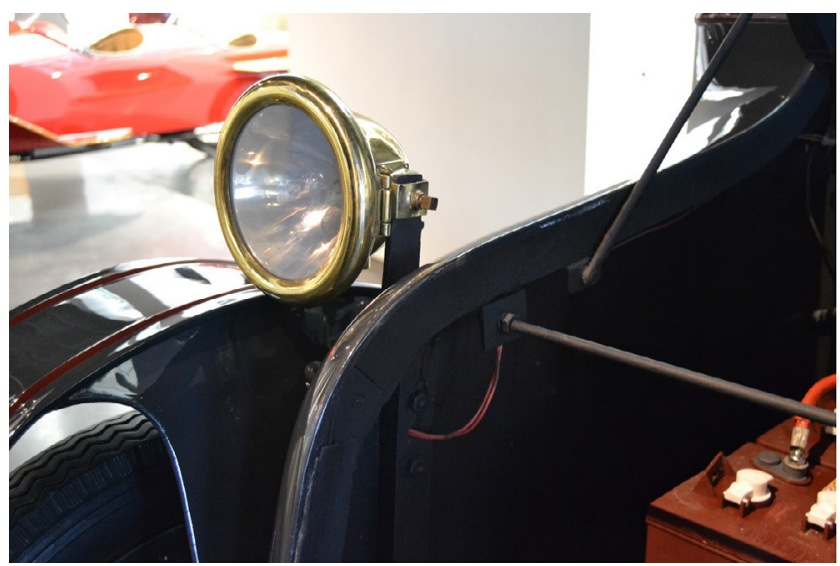

Fig. 5. Faro delantero derecho del JJJG

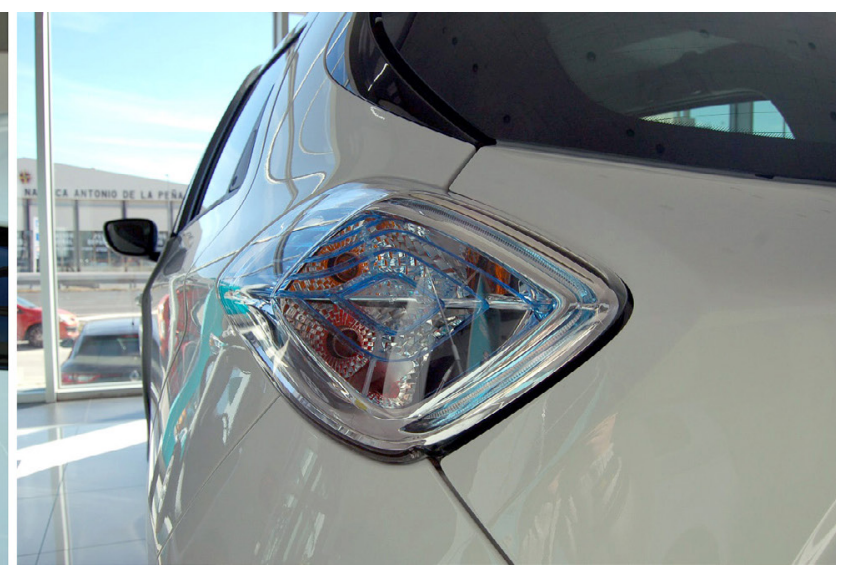

Fig. 4. Faro trasero izquierdo del Zoe. JJJG.

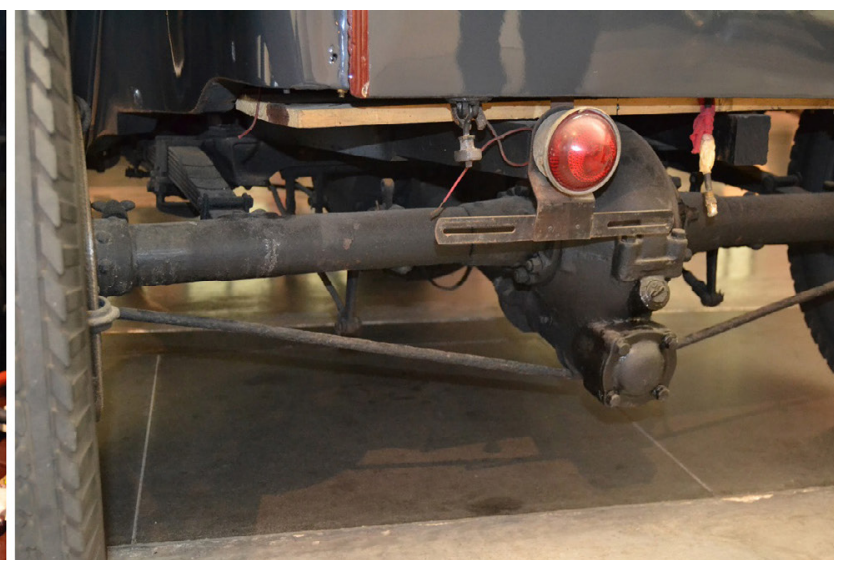

Fig. 6. Faro trasero del Model 22. JJJG

5 Cuadrado, D. (22 de enero de 2019) Supercomparativa. Hyundai Kona-Nissan Leaf-Renault Zoe. Autopista. Volumen 3085, pp. 12-23. 
Entre los años 1915 y 1923 se fabricaron 4000 unidades del Model $22^{6}$ y, en concreto, durante el año 1915 se construyeron 1000 unidades, que en 1916 fueron 1500 más. Se aprecia un incremento de las ventas de este modelo al inicio, y cómo la consideración de un vehículo eléctrico iba en aumento. Aunque este interés se mantuvo por poco tiempo, ya que los eléctricos empezaron a dejar de despertar interés. El problema que hubo hace un siglo en las baterías con respecto a su peso y potencia suministrada, hicieron por entonces desechar esta tecnología eléctrica ${ }^{7}$, y eso sin contar las autonomías de las mismas, siguen existiendo hoy día, aunque con vehículos como el Zoe se atisba un nuevo futuro para esta tecnología.

La velocidad que alcanzaba la versión roadster era de $30,5 \mathrm{~km} / \mathrm{h}$ y en la versión cupé de casi $25 \mathrm{~km} / \mathrm{h}$, velocidad superior aunque similar a la de un autocamión de una tonelada de capacidad de carga, siendo estas de 24 $\mathrm{km} / \mathrm{h}$ para la versión ómnibus y $19 \mathrm{~km} / \mathrm{h}$ para la versión de carga ${ }^{8}$.

El Model 22 cuenta con una serie de baterías en la parte delantera y trasera (figs. 7 y 8 respectivamente), que daban al vehículo una autonomía entre 96 y 120 kilómetros. Para evitar el problema del tiempo de espera en la recarga de las baterías, en el año 1918 Milburn idea una pequeña estructura mediante rodillos, con la que se extraía la batería descargada y se introducía la cargada, lo que supuso que el tiempo de espera del cambio de baterías fuese muy similar al del repostaje de gasolina en un motor de combustión.

El Milburn Model 22 de las imágenes usa baterías actuales, y con 103 años sigue funcionando y es capaz de desplazarse por sí mismo. Muy buen trabajo hicieron en su diseño y fabricación para que se pueda contar con una joya como ésta a día de hoy y tenerla en el magnífico estado en que se encuentra.

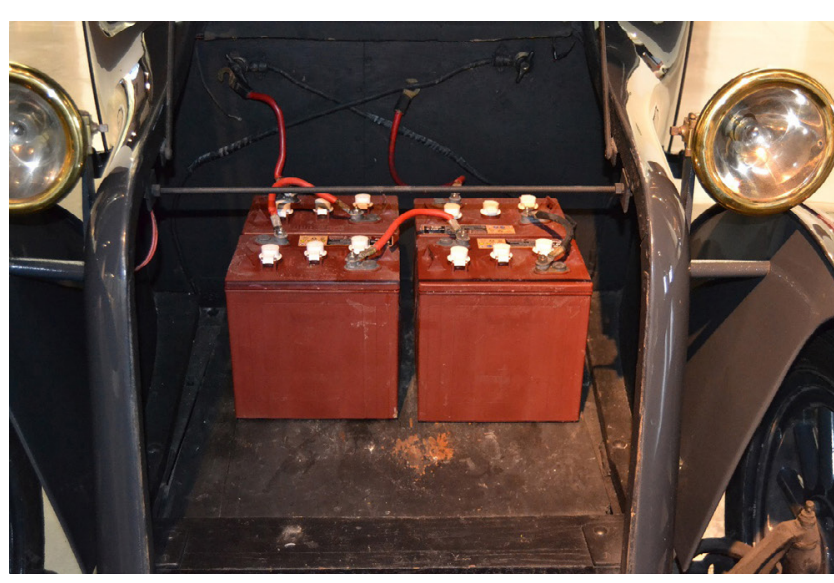

Fig. 7. Baterías en la parte delantera del Milburn (son baterías actuales las que tiene este Model 22 a día de hoy). JJJG.

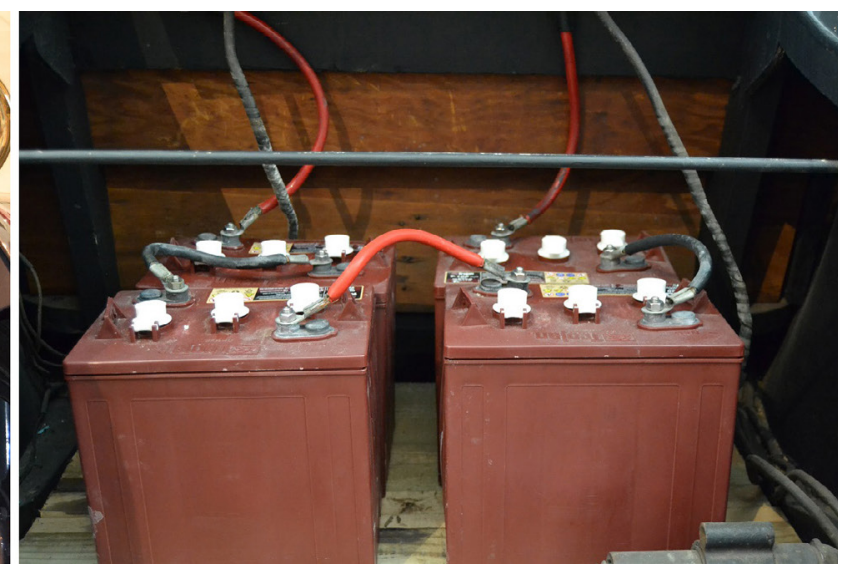

Fig. 8. Baterías en la parte trasera del Milburn (también son baterías actuales). JJJG.

Evidentemente, cuando se habla de la autonomía del Milburn no corresponde a datos del ciclo europeo NEDC (New European Driving Cycle) o del nuevo protocolo WLTP (Worldwide Harmonized Light-Duty Vehicles Test Procedure) por el que se rigen ahora los vehículos, ya que el protocolo NEDC se usa desde los años 90, el WLTP desde septiembre de 2017, y el Model 22 es de 1916.

\footnotetext{
6 Magalhães, J. (2018). Museo Automovilístico y de la Moda. El automóvil como obra de arte. Málaga, España: PR Grafis, p. 57.

7 Zerolo, M. (1926). Manual Práctico de Automovilismo. París, Francia: Casa Editorial Garnier Hermanos, p. 7.

8 W. Pagé, Víctor. (1924). Autocamiones Modernos de Gasolina y Eléctricos. Guía Práctica del Automovilista. Barcelona, España: Editorial Labor, S.A., p. 810.
} 
En cambio el Zoe si se rige por el WLTP, que usa la innovación como forma de relacionarse con el mundo ${ }^{9} \mathrm{y}$ tratar de obtener un mundo mejor. Este modelo de Renault, con la batería de iones de litio con una capacidad de $41 \mathrm{kWh}$, consigue una destacable autonomía real de 300 kilómetros, que también coincide con la obtenida por el protocolo WLTP ${ }^{10}$. Con la incorporación de esta batería, al modelo Zoe, se consigue que el vehículo eléctrico ya no quede limitado a moverse por el centro urbano, sino que posibilita trayectos interurbanos. Ello permite que la opción de tener un modelo eléctrico, en vez de uno de combustión interna, sea mucho más real, con la consiguiente mejora en la sostenibilidad del medio ambiente por la disminución de los gases contaminantes. Evidentemente, esto conlleva un aumento de la percepción positiva de Renault como empresa fabricante de vehículos por parte de la población, que pueden ver con buenos ojos la adquisición de un modelo "verde" de esta marca para contribuir a la mejora del entorno y propiciar un mejor medio ambiente.

Siguiendo con la comparación del diseño de estos dos vehículos, hablando ya específicamente del diseño interior, en el caso del Milburn se podía comparar con un pequeño salón de una casa (fig. 9), siendo los asientos delanteros abatibles (fig. 10) y enfrentados a los traseros donde se sienta el conductor del piloto. Se puede entender que estos asientos delanteros estaban diseñados para niños, pues si se sentaban dos adultos delante la visión del conductor podría llegar a ser nula.

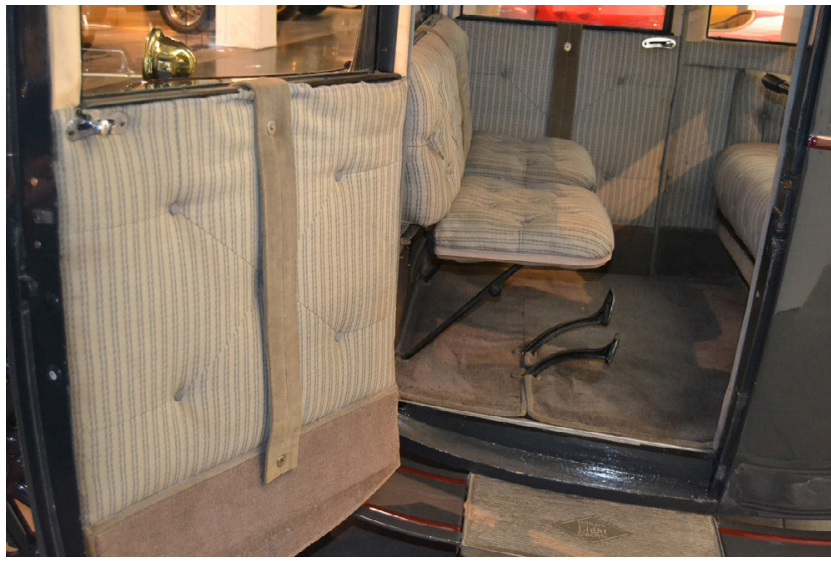

Fig. 9. Interior del Model 22. JJJG

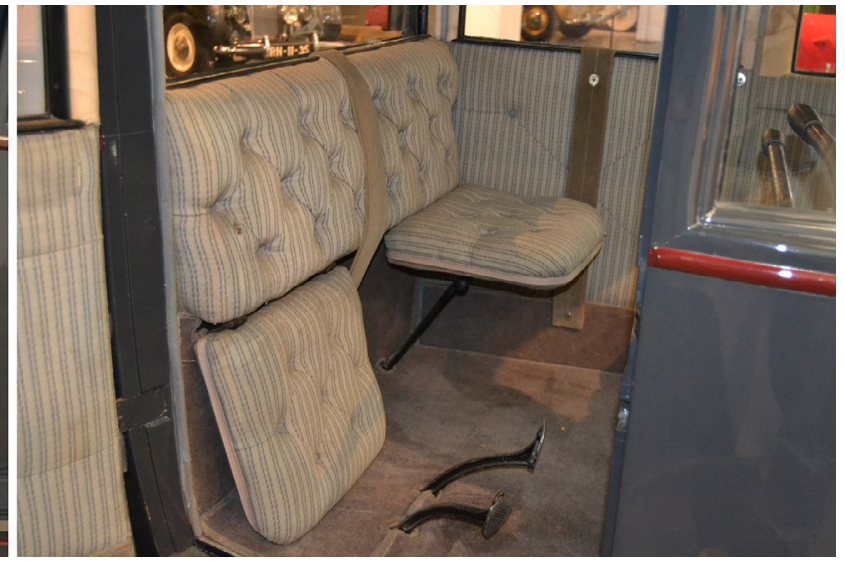

Fig. 10. Interior del Model 22 con uno de los asientos delanteros abatido y el otro no. JJJG

El interior del Zoe es completamente actual, como se comprueba en sus asientos delanteros (fig. 11) y traseros (fig. 12), siendo muy funcionales a la vez que cómodos, logrando en su conjunto muy buena habitabilidad y una gran capacidad en el maletero de 370 litros $^{11}$.

En el diseño de los cuadros de mando se aprecia perfectamente el salto tecnológico entre ambos modelos; el del Milburn (fig.13) la única información que proporciona es el voltaje y amperios disponibles en la batería. El cuadro del Zoe (fig. 14), con una pantalla TFT, posee toda la información que un usuario desee y pueda necesitar, ampliada con otra pantalla en la zona central (fig. 15), desde dónde se controlan muchas de las opciones que tiene el Zoe.

\footnotetext{
9 Rossi, M. (2016). Migliorare i comportamenti e le relazioni sociali mediante l'innovazione dei processi partecipativi driven design. I+Diseño, 11. 110-117.

10 Murias, D. (13 de septiembre de 2018. Actualizado 15 de septiembre de 2018). Xataka. Weblogs. Recuperado de https://www. xataka.com/vehiculos/wltp-coches-electricos-autonomia-real-no-va-a-ser-que-anunciaba-fabricante

11 Vitoria, L.M. (febrero 2019). Comparativa. Renault Zoe-Smart Forfour. Autovía. Volumen 322, pp. 56-59.
} 


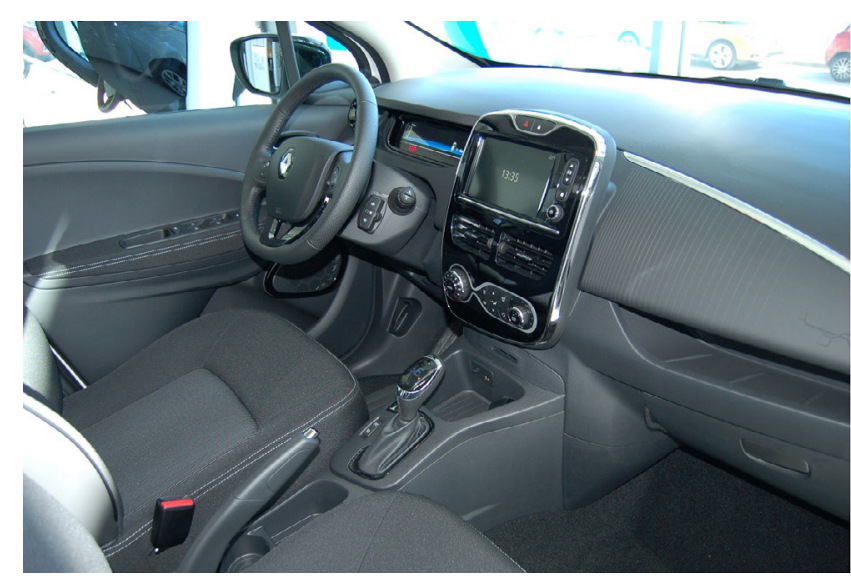

Fig. 11. Asientos delanteros del Zoe. JJJG

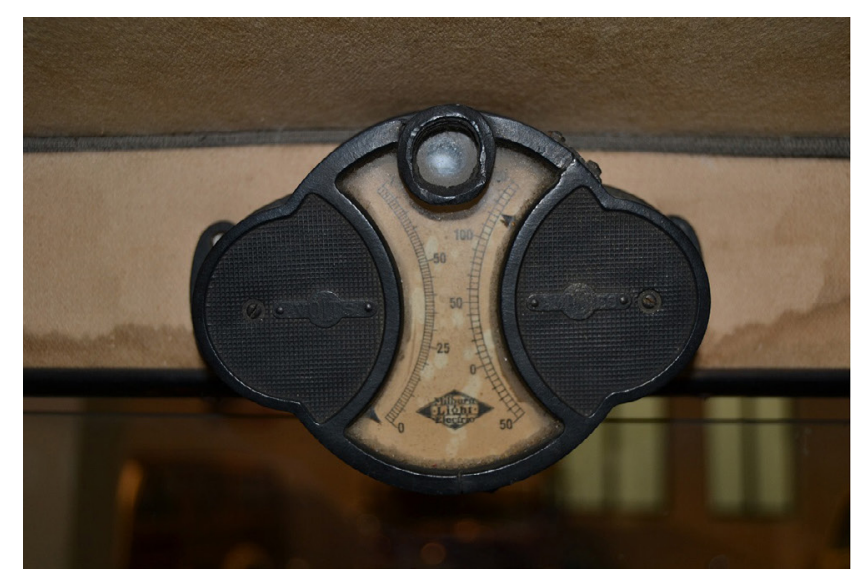

Fig. 13. Cuadro de mandos del Milburn. JJJG

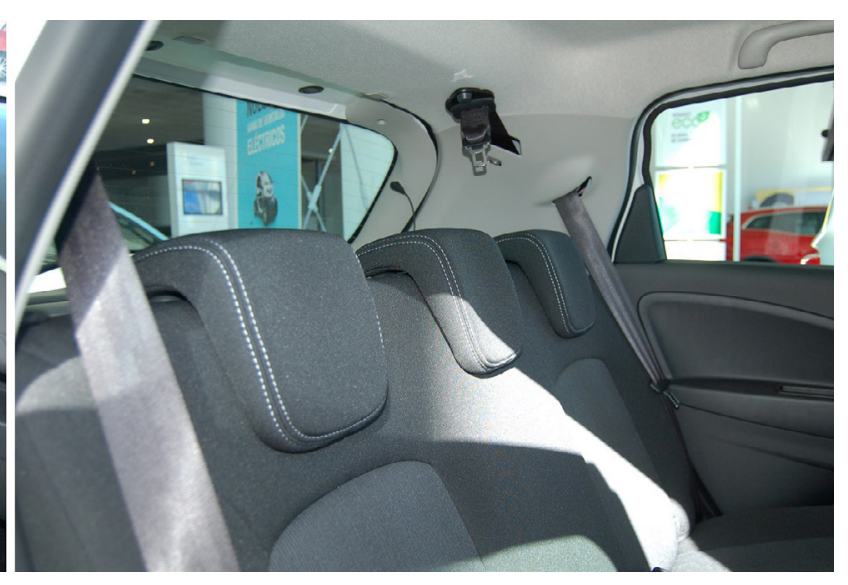

Fig. 12. Asientos traseros del Zoe. JJJG

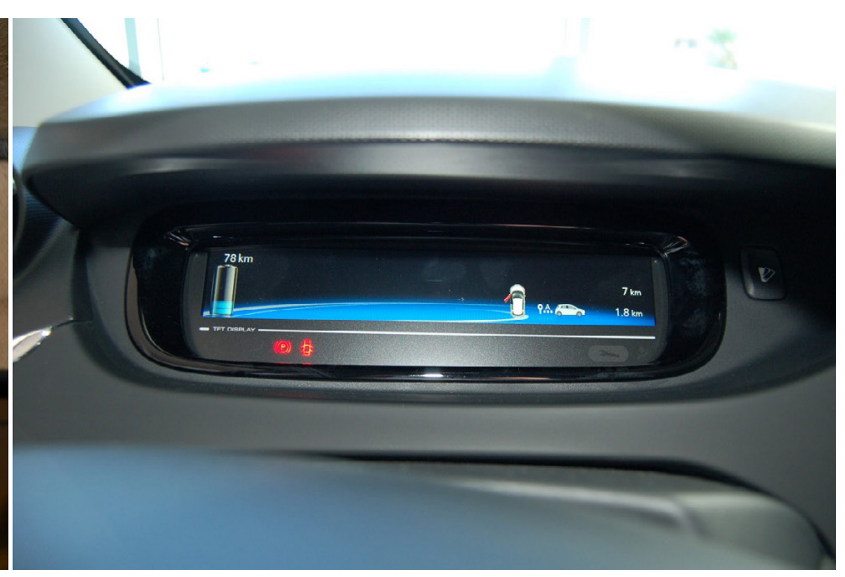

Fig. 14. Cuadro de mandos TFT del Zoe. JJJG

En la fig. 15 se aprecia, a la izquierda de la pantalla, el botón para activar el motor o cortar el contacto del Zoe, poniendo el vehículo en estado de "ready" para que el sistema del coche esté listo y comience a funcionar.

El Model 22 ya contaba con algo parecido, pues disponía de un interruptor principal ubicado en el reposabrazos izquierdo (fig. 16), para que el conductor lo tuviera a mano. Este interruptor tenía tres posiciones: para activar el motor y poder mover el vehículo, desconectar el motor para estacionar o poner en carga las baterías.

En los vehículos eléctricos, son destacables diferentes ventajas; entre estas se encuentran las de tener una marcha suave en carretera siendo a la vez más silenciosos que un vehículo de combustión interna, y una mayor facilidad para el conductor de regular la fuerza de tracción y la velocidad del vehículo ${ }^{12}$. 


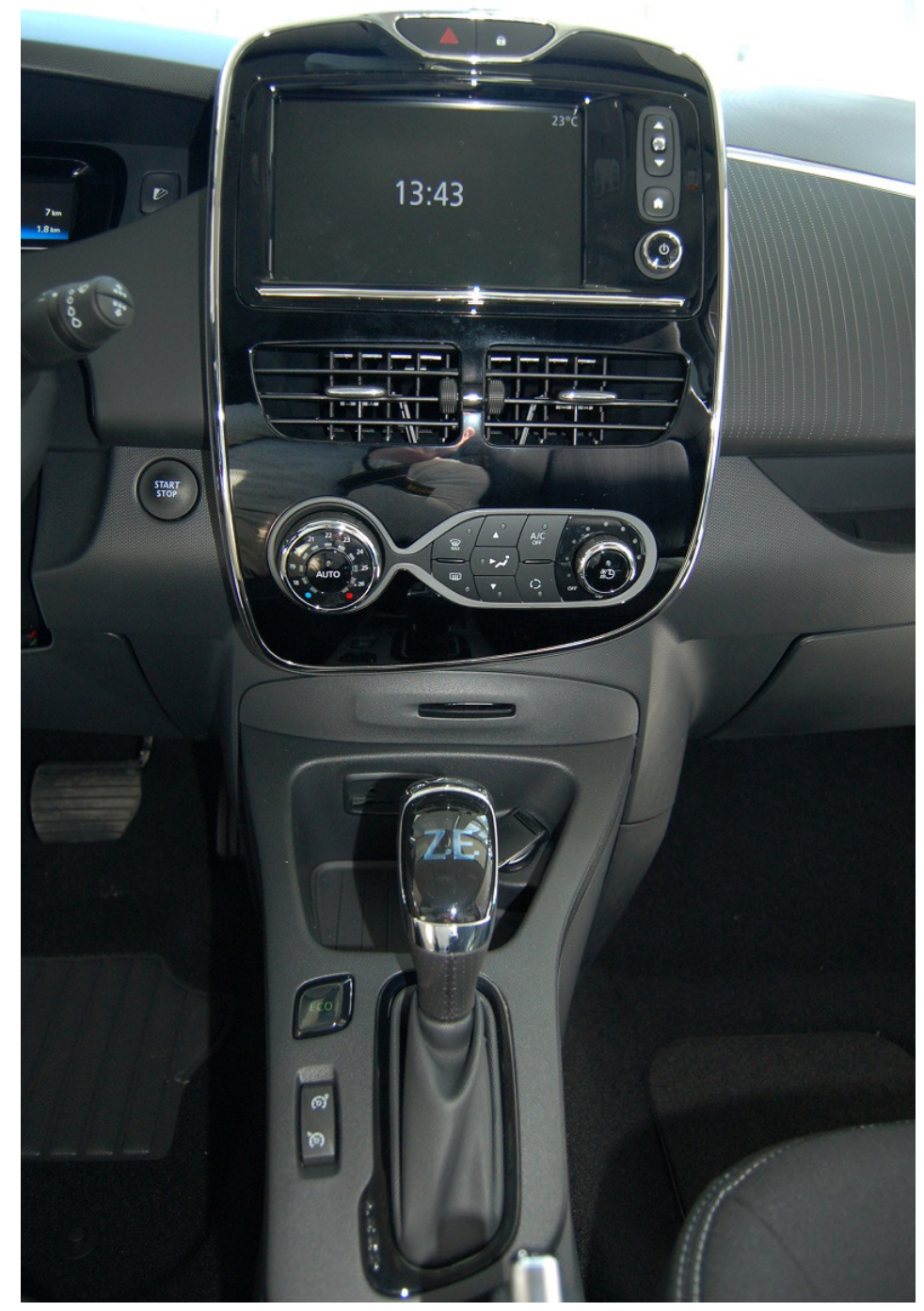

Fig. 15. Pantalla y mandos centrales del Renault Zoe. JJJG

Otra característica a destacar del Model 22 son sus mandos para el control y la dirección del vehículo (fig. 17). Son dos palancas, una larga y otra más corta, que cuando los usuarios querían entrar al vehículo se colocaban estos mandos en posición vertical, para que una vez sentados, el conductor los coloque en posición horizontal para comenzar a rodar con el vehículo. La palanca larga controlaba la dirección, si se desplaza hacia delante el vehículo giraba hacia la izquierda, y si se traía hacia atrás el vehículo giraba hacia la derecha. La palanca corta parte de la posición de neutralidad, y si se movía hacia delante ${ }^{13}$ había cuatro muescas que correspondían con las cuatro velocidades que tiene el Model 22. Otras dos velocidades eran para la marcha atrás ${ }^{14}$ cuando el conductor accionaba la palanca hacia sí mismo, pasando por la zona neutra.

13 AA.VV. "Instruction Book" Model 27 L. The Milburn Wagon Co. Toledo, Ohio, p. 11.

14 Magalhães, J. Op. Cit., p. 57. 


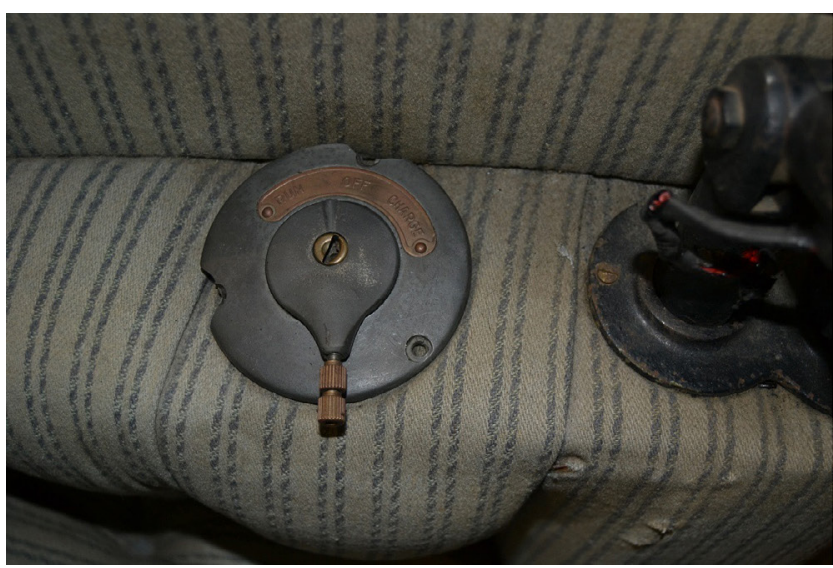

Fig. 16. Interruptor de mando del Milburn Model 22. JJJG

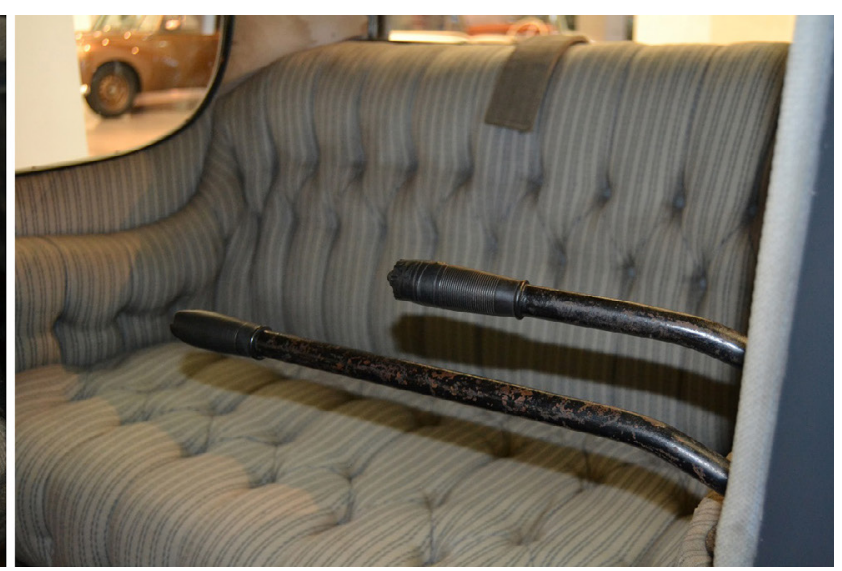

Fig. 17. Mandos del Model 22 para la dirección y control del vehículo. JJJG

Cuando se quería ir hacia delante era necesario que la palanca encajara bien en cada muesca, y si se quería usar el freno eléctrico sencillamente se llegaba al punto neutro y se tiraba de la palanca hacia atrás.

Para poder engranar la marcha hacia atrás, era necesario llevarse la palanca hacia atrás todo lo posible; entonces el conductor pisaba un pequeño pedal con el talón, que se aprecia en la fig. 18, y en ese momento se podía engranar la primera marcha hacia atrás, o la segunda, según fuera necesario. Un detalle es que en la palanca de control también se encuentra un pequeño pulsador para la bocina ${ }^{15}$.

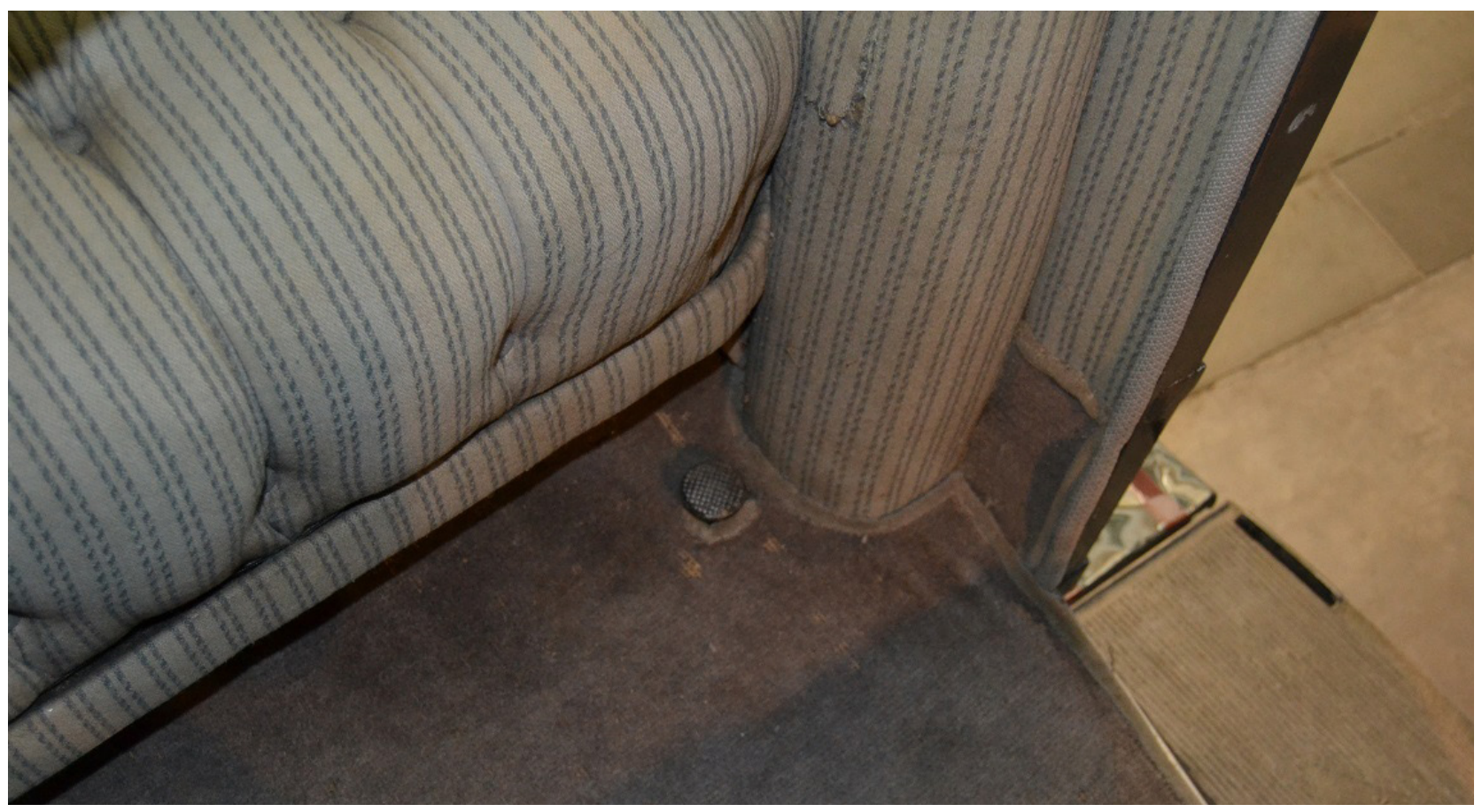

Fig. 18. Muy cerca del asiento, en el suelo, se encuentra el pedal con forma circular que permite engranar las marchas hacia atrás. JJJG

15 AA.VV. "Instruction Book" Model 27 L. Op. Cit. p. 11. 
Al propietario del Model 22 le avisaban que no usara la marcha primera, hacia delante o hacia atrás, durante más de 10 minutos seguidos, detalle que no le ocurre al Zoe ni a los vehículos eléctricos actuales ${ }^{16}$.

Otra enorme diferencia que se encuentra entre el Zoe y el Milburn está en el apartado de los frenos. En ambos existe el freno eléctrico para la circulación normal, pero cuando se necesita más potencia de frenada el Zoe cuenta con unos discos delanteros de $258 \mathrm{~mm}$ de diámetro (fig. 19), y en los traseros recurren a un sistema de freno de tambores ${ }^{17}$ (fig. 20), que cumplen con su cometido de manera sobresaliente.

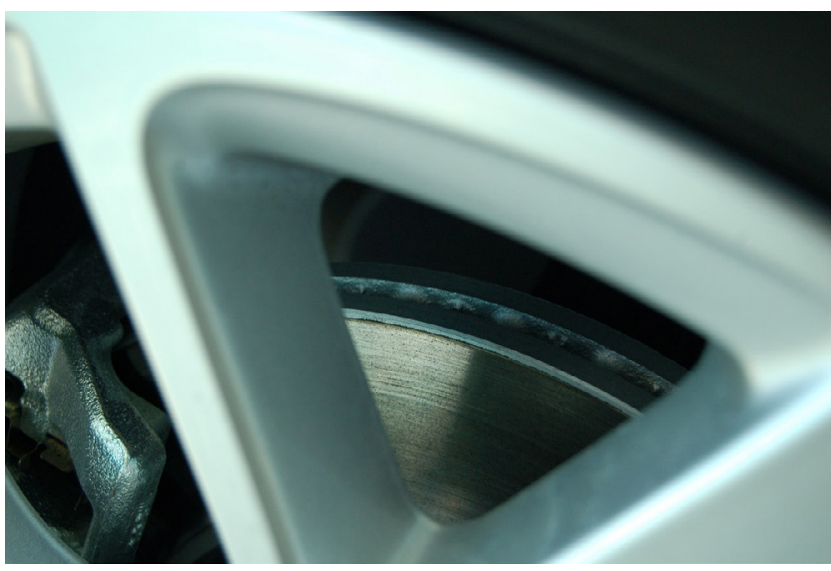

Fig. 19. A través de las llantas de aleación ligera del Zoe se ve el disco de freno y su pinza. JJJG

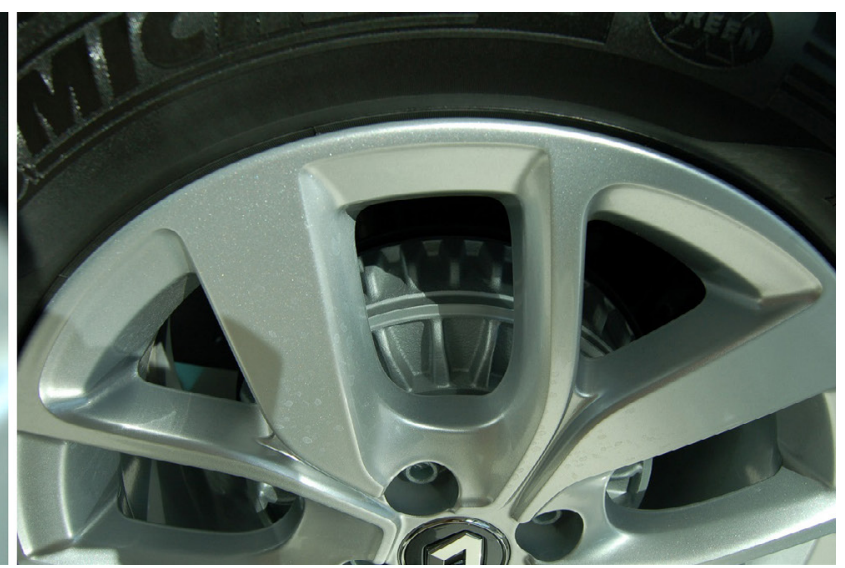

Fig. 20. Aquí se puede apreciar el freno de tambor del Zoe, a través de la llanta de aleación en cada rueda trasera. JJJG

Para frenar el Milburn disponía, además de como se ha indicado, del freno eléctrico, de dos pedales (figs. 9 y 10). El pedal izquierdo era el encargado de activar el freno de expansión interna ubicado dentro de la rueda trasera a través del mecanismo que se observa debajo del anclaje derecho del muelle cantilever ${ }^{18}$ (ballestas) en la fig. 21, debiéndose usar este tipo de freno solo en caso de emergencia o para dejar estacionado el coche, lo que viene a ser el freno de mano actual que por ejemplo lleva el Zoe.

El pedal derecho era el encargado de activar el freno de contracción externa también en las ruedas traseras ${ }^{19}$ (fig. 22). Este freno era el usado normalmente para las situaciones cotidianas de conducción. Este pedal servía a su vez para soltar el freno de bloqueo que se activaba con el pedal izquierdo, al liberar el trinquete que dicho pedal accionaba.

Para tratar de prevenir accidentes, el Milburn hacía sonar la bocina si el freno de estacionamiento estaba activado y la palanca de control no estuviera en la posición de neutro. La marca también hacía hincapié en que el freno de emergencia (el del pedal izquierdo) no se usara a no ser que fuera imprescindible, y si fuera el caso, que la palanca de control se llevara al neutro lo más rápidamente posible, a la vez que se pisaba el pedal. Otra buena práctica necesaria, que la marca recordaba, era que al dejar el coche estacionado se debía poner el interruptor de la fig. 16 en la posición "off", y además se debía dejar pisado el pedal del freno izquierdo ${ }^{20}$.

16 AA.VV. "Instruction Book" Model 27 L. Op. Cit. p. 13.

17 Vitoria, L.M. (febrero 2019). Op. Cit., p. 56-59.

18 Zerolo, M. Op. Cit., p. 201.

19 AA.VV. "Instruction Book" Model 27 L. Op. Cit. p. 13.

20 AA.VV. "Instruction Book" Model 27 L. Op. Cit. p. 14. 


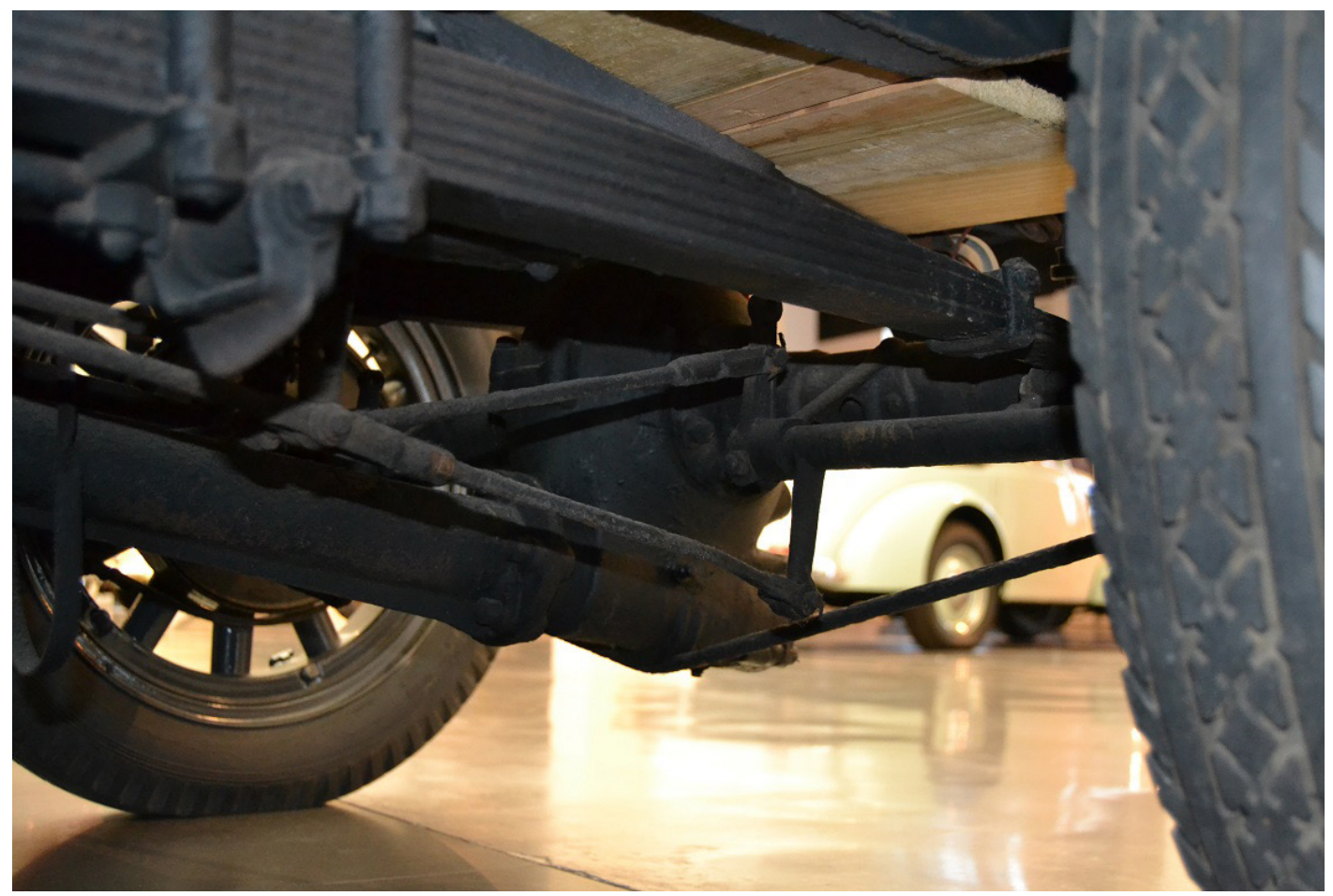

Fig. 21. Mecanismo para el sistema de frenado de expansión interna del Model 22. JJJG

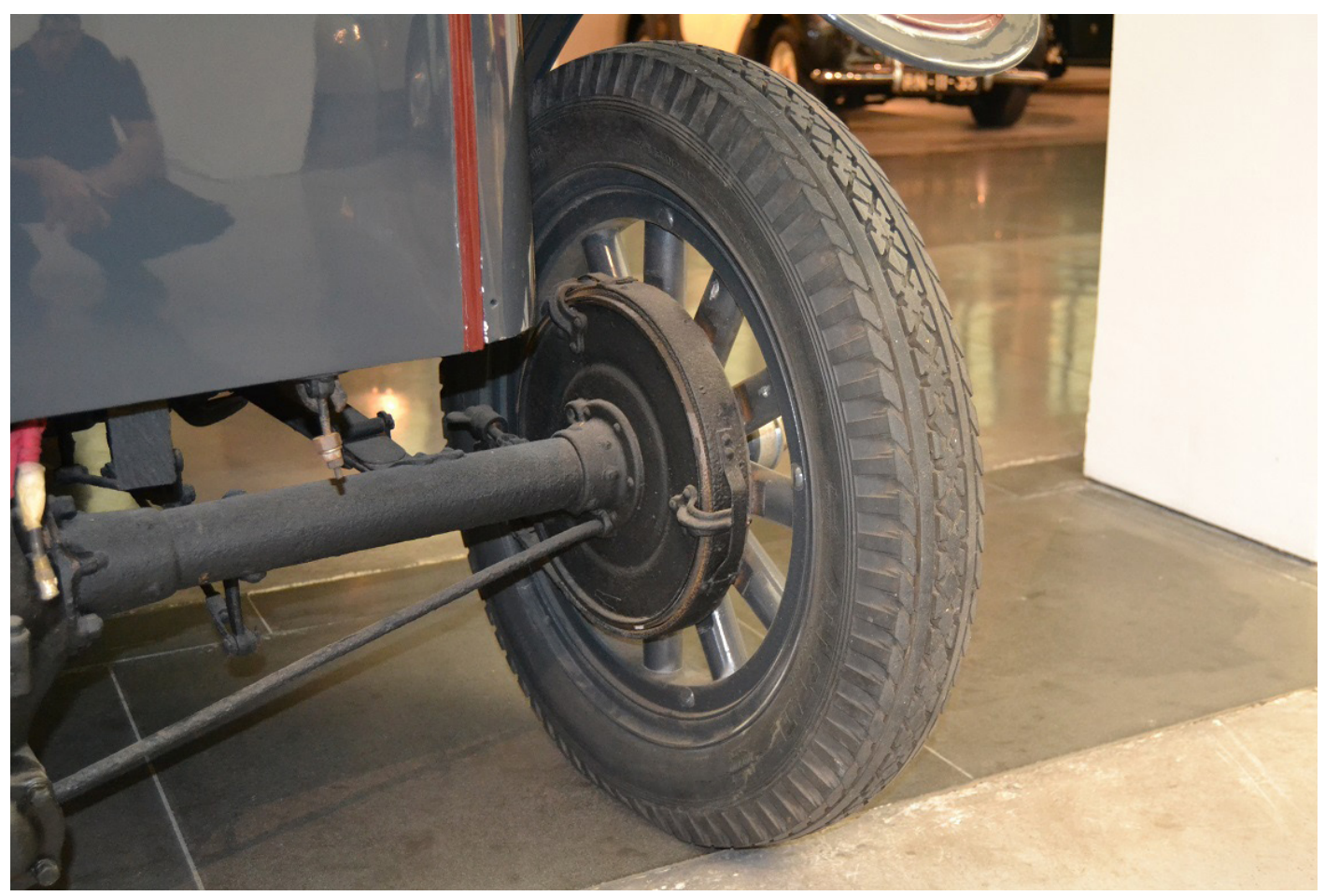

Fig. 22. Mecanismo de activación del freno de contracción externo accionado mediante el pedal derecho. JJJG 


\section{Conclusiones}

Cuando se revisan las características del Renault Zoe en cuanto a sostenibilidad, versatilidad, funcionalidad y por supuesto en diseño, entiende perfectamente porque es el actual número uno en ventas de España en vehículos eléctricos. Esta apuesta de Renault en los vehículos eléctricos hace indudablemente que la visión que se tiene sobre la marca mejore de manera proporcional a su preocupación por el medio ambiente, mejorando la Responsabilidad Social Corporativa de la empresa.

Los datos que manejan instituciones como la Agencia Europea del Medio Ambiente (AEMA) en su "Air Quality in Europe 2018" o la Organización Mundial de la Salud en sus "Guías de calidad del aire de la OMS relativas al material particulado, el ozono, el dióxido de nitrógeno y el dióxido de azufre" demuestran la importancia de acometer de una manera firme y decidida la apuesta por una movilidad sostenible, ya que de ello depende gran parte de la salud de las personas, sobre todo en las urbes, y de que exista un equilibrio sostenible en el planeta.

Si cualquier persona se pone a estudiar un vehículo que se construyó hace un siglo, espera encontrar tecnología obsoleta. Pero la sorpresa es cuando resulta que, en su esencia, funciona casi como un vehículo eléctrico actual, y la autonomía que ya tenía por aquel entonces estaba entre 96 y 120 kilómetros, cuando las ciudades de antaño eran bastantes más pequeñas que las de ahora y, en consecuencia, todo estaba más cerca y suponía un gasto menor de la batería. Salvando las distancias de materiales y de comodidad, la tecnología de este Milburn podría ser usada en coches eléctricos actuales, y seguro que daría un rendimiento digno a más de un usuario. Hay que tener en cuenta que el Model 22 era el vehículo eléctrico más eficiente, barato y a la vez el más ligero de la época.

Seguramente, si se hubiera seguido avanzando en aquella tecnología eléctrica para los vehículos, en estos 103 años que han pasado, la tecnología en vehículos eléctricos sería impresionante, ya sea en la duración de sus baterías, en la potencia suministrada, en la gestión de la energía, etc. Lo que hace pertinente la siguiente pregunta: ¿Cómo sería la tecnología en este campo si hace un siglo si una empresa hubiese seguido investigando y mejorando aquella tecnología en los vehículos eléctricos? O esta otra pregunta: ¿Qué habría ocurrido si se hubiese continuado el desarrollo de la tecnología del Milburn Model 22 y cómo sería hoy, 103 años después? Estas dos preguntas jamás podrán ser resueltas, pero se podría intuir que a los motores de combustión, mayoritarios en la actualidad, les habría ganado la partida el motor eléctrico.

A pesar de todo, no podemos negar la evidencia de que necesitamos una movilidad más sostenible, más limpia, sobre todo en las ciudades, porque ahí es donde más sufren por contaminación los ciudadanos. A través del propósito de la mejora de la Responsabilidad Social Corporativa y la repercusión positiva de ésta sobre las empresas o instituciones públicas que buscan mejorarla, se puede conseguir impulsar una movilidad más racional, por ejemplo incentivando el uso del vehículo eléctrico, el del híbrido o el del híbrido eléctrico enchufable por decir algunas tecnologías de las disponibles para desplazarse por la ciudad. Esto, a su vez, requeriría una mejor instalación de puntos de recarga (para aquellos que se pudieran enchufar a la red eléctrica) que animaran al usuario a comprar ese vehículo sin miedo a no poder realizar los kilómetros de autonomía que se deseen, ya que si lo comparamos con los vehículos tradicionales de combustión interna, éstos últimos poseen más autonomía y a la vez una red muy extensa de gasolineras que hace difícil pensar en no llegar al destino que se quiera pudiendo repostar. 


\section{Bibliografía.}

AA.VV. "Instruction Book" Model 27 L. The Milburn Wagon Co. Toledo, Ohio.

Boehe, D.M. y Cruz, L.B. (2010). Corporate social responsibility, product differentiation strategy and export performance. Journal of Business Ethics, Vol. 91 No. 2, pp. 325-346.

Cuadrado, D. (22 de enero de 2019) Supercomparativa. Hyundai Kona-Nissan Leaf-Renault Zoe. Autopista. Volumen 3085, pp. $12-23$.

Magalhães, J. (2018). Museo Automovilístico y de la Moda. El automóvil como obra de arte. Málaga, España: PR Grafis.

McWilliams, A. y Siegel, D. S. (2011). Creating and capturing value: Strategic corporate social responsibility, resource-based theory, and sustainable competitive advantage. Journal of Management, 37 (5). 1480-1495.

Murias, D. (13 de septiembre de 2018. Actualizado 15 de septiembre de 2018). Xataka. Weblogs. Recuperado de https://www.xataka.com/vehiculos/wltp-coches-electricos-autonomia-real-no-va-a-ser-que-anunciabafabricante

París Eguilaz, H. (1941). Un Nuevo Orden Económico. Madrid, España: Ediciones FE.

Rossi, M. (2016). Migliorare i comportamenti e le relazioni sociali mediante l'innovazione dei processi partecipativi driven design. I+Diseño, 11.110-117.

Vitoria, L.M. (febrero 2019). Comparativa. Renault Zoe-Smart Forfour. Autovía. Volumen 322, pp. 56-59

W. Pagé, Víctor. (1924). Autocamiones Modernos de Gasolina y Eléctricos. Guía Práctica del Automovilista. Barcelona, España: Editorial Labor, S.A.

Zerolo, M. (1926). Manual Práctico de Automovilismo. París, Francia: Casa Editorial Garnier Hermanos. 